\title{
Méthodes d'étude des transports solides en rivières, estuaires et zones côtières
}

\author{
P. Monadier \\ Ingénieur en Chef des Ponts-et-Chaussées, \\ Chef du Service Technique Central des Ports maritimes et des Voies navigables
}

La connaissance des évolutions sédimentaires constitue toujours un élément essentiel pour la prise de décision concernant la gestion, la conservation ou l'aménagement des domaines en équilibre, la plupart du temps fragile, que constituent les rivières, les estuaires et le littoral.

Les principaux problèmes à résoudre apparaissent pouvoir être résumés comme suit :

- évolution naturelle des zones fluviales, estuariennes ou côtières considérées. en l'absence de toute intervention humaine.

- conséquences quantitatives sur les plans des érosions. dépôts. transports sédimentaires. de décisions envisagées concernant leur gestion, voire leur aménagement.

- conséquences qualitatives, sur le plan des caractéristiques physico-chimiques des eaux, de ces mêmes décisions.

\section{Les Méthodes}

Plusieurs méthodes sont utilisables pour aborder l'étude de ces problèmes.

Pendant longtemps, ils ont été, malgré leur importance. soit ignorés, soit traités, à partir de connaissances pragmatiques et incomplètes, sous la forme d'études sur plans faisant davantage appel à l'intuition et au bon sens qu'à un corps cohérent de connaissances techniques.

Depuis une quarantaine d'années, la compréhension des phénomènes physiques en cause s'est substantiellement améliorée et le recours à des modèles réduits physiques est devenu un moyen fiable et assez précis d'étude des différents types de problèmes posés. Sous leur forme moderne. ces modèles présentent des atouts très importants :

- sous réserve qu'ils soient correctement conşus et exploités. représentation convenable de la plupart des phénomènes en cause.

- appréhension assez fine des évolutions naturelles et des conséquences des décisions envisagées.

- caractère démonstratif affirmé à l'égard des autorités et bureaux d'études appelés à connaître des études correspondantes.

Mais leurs inconvénients sont néanmoins réels :

- délais importants requis pour leur mise en auvre,

- couts élevés de la réalisation des modèles et de leur. exploitation.
- limitation, en raison de ces coûts, des emprises physiques des modèles, alors qu'il serait souvent souhaitable. pour appréhender l'ensemble des phénomènes en cause, de prendre en compte des zones suffisamment étendues,

- limitation. pour les mêmes raisons, de la durée des évolutions examinées.

- limitation inévitable du nombre et des caractéristiques des solutions susceptibles d'être examinées lors de l'étude de projets d'aménagement.

L'amélioration de la compréhension des phénomènes physiques en cause, la maîtrise obtenue des modèles réduits physiques, les progrès généraux obtenus en matière de modélisation numérique et de performances des ordinateurs et le souci d'améliorer les études, tout en en limitant sévèrement le cô̂t, ont provoqué l'émergence des modèles sédimentologiques numériques.

Leurs avantages sont a priori évidents :

- rapidité de mise en auvre,

- moindre coût d'exploitation des modèles, sinon du développement des codes.

- extension possible sans difficultés importantes, de l'emprise physique des modèles et de la durée de simulation des évolutions.

- multiplication possible. à des côtrs modérés, des solutions d'aménagements susceptibles d'être examinées et des tests de sensibilité susceptibles d'être conduits.

Mais leurs inconvénients restent importants :

- connaissance encore imparfaite des phénomènes physiques susceptibles d'être pris en compte, et donc limitation de leurs possibilités d'intervention.

- simplification obligatoire des phénomènes, ainsi que des impacts des décisions et des aménagements susceptibles d'être valablement représentés, en raison des coûts de calcul.

\section{Modèles numériques ou modèles physiques}

En fait, modèles physiques et numériques ne sont plus guère en opposition, mais, dans la plupart des applications pratiques, plutôt complémentaires. La rapide analyse donnée ci-dessus des avantages et des inconvénients de chacun de ces types d'outils suffit à le mettre en évidence. Ils permettent en pratique de traiter soit des problèmes 
différents, soit différents aspects d'un même problème.

Très schématiquement :

- les modèles numériques sont les meilleurs pour effectuer des études de dégrossissage pouvant porter sur de vastes zones et de longues durées, lorsque les configurations géométriques et hydrographiques et les phénomènes en cause ne sont pas trop compliqués; ils permettent d'examiner rapidement différents scénarios d'évolution, notamment en cours de projets d'aménagements ;

- les modèles physiques sont préférables pour affiner les études, sur des zones restreintes et des durées pas trop longues, quelque soit la complexité des conditions géométriques, hydrographiques, hydrodynamiques et sédimentologiques.

Sur le plan de la progression des connaissances et des moyens d'études, les deux approches s'enrichissent l'une l'autre :

- les mesures in situ et l'expérimentation sur modèle physique contribuent à l'amélioration des schémas et formules à introduire dans les modèles numériques; - les modèles numériques, d'une part contribuent à mettre en évidence l'insuffisance des connaissances physiques et provoquent les recherches nécessaires pour combler les lacunes constatées ou seulement compléter les formulations existantes, d'autre part permettent de tester certaines lois physiques expérimentales, sous la forme de simulations et de validations économiques.

\section{Les thèmes détaillés dans ce numéro}

Les communications reprises dans le présent numéro de La Houille Blanche donnent un panorama, certes incomplet, mais tout de même assez riche, des travaux récemment conduits, au plan français, en matière de modélisation des phénomènes fluviaux, estuariens et littoraux. Ils couvrent une gamme de problèmes s'étendant de l'observation et de l'expérimentation in situ à des recherches de pointe sur certains phénomènes mal connus et la possibilité de leur modélisation numérique.

La communication de F. LEVOY, O.MONFORT, H. ROUSSET et C. LARSONNEUR (Laboratoire de géologie Marine de l'Université de Caen) relative à la modélisation des transports sédimentaires sur les plages macrotidales et à leur application sur la côte ouest du Cotentin rend compte des résultats provisoires d'une opération de vaste envergure engagée depuis plusieurs années sur cette portion du littoral. Après avoir rappelé le cadre physique de la côte ouest du Cotentin (conditions hydrodynamiques et morphologie des plages), brièvement présenté les deux modèles numériques pour le calcul des facteurs hydrodynamiques responsables du transport sédimentaire (modèle de courant DUCHESS et modèle de houle HISWA) et rappelé les différentes formules disponibles pour l'estimation du transport sédimentaire sous l'action simultanée des houles et des courants, tant par charriage qu'en suspension, elle s'attache à proposer le choix d' une formulation adaptée au contexte local. Ce choix, effectué sur la base de campagnes de mesures in situ importantes, utilisant la technique des traceurs fluorescents et des pièges à sédiments multidirectionnels, aboutit à la formule de vaN
RIJN, qui donne les résultats les plus satisfaisants, avec néanmoins une surestimation des transports sédimentaires dans $70 \%$ des cas environ, les résultats étant meilleurs pour des conjonctions associant des houles fortes et des courants faibles que pour toutes situations de houles avec des courants plus élevés. Cette formule permet aussi de rendre compte de la direction du transport.

La communication de C. VILLARET $(L N H)$, relative à l'étude expérimentale du transport sédimentaire sous l'action de la houle et du courant, rend compte des essais en canal réalisés par le $\mathrm{LNH}$, dans le cadre du projet européen «MAST G8M Morphodynamics" pour étudier le transport de sables fins sous l'action combinée de la houle et des courants.

Les conclusions obtenues sont les suivantes:

- Dans le cas d'un écoulement permanent sur fond ridé asymétrique, la turbulence dans la couche limite de fond peut être modélisée en introduisant un coefficient de rugosité équivalente $k s$, dont la valeur est proportionnelle à la hauteur des rides. Les dimensions moyennes des rides à l'équilibre ont tendance à augmenter en fonction du courant dans la plage de régimes étudiés. Le transport total est très faible et n'a pas pu être estimé avec précision. - Les processus de transport en houle et houle et courant combinés sont gouvernés par la génération et l'intensité des tourbillons formés à chaque demi-période par les phénomènes de décollement de la couche limite responsables de la mise en suspension du matériau. Ces processus importants ne peuvent être traités par les modèles de couche limite classiquement utilisés pour des écoulements permanents en régime hydrauliquement rugueux. Dans les conditions des essais, en l'absence de courant, le transport moyen est dans le sens de propagation de la houle, alors qu'un courant de même sens que la houle inverse le sens du transport moyen.

D'autres résultats expérimentaux sont rapportés par MM. D. AELBRECHT et G. CHABERT d'HIËRES. Ils ont mis en évidence l'effet de "pompage d'Ekman " qui consiste en une rectification à la côte des effets de Coriolis et crée une dérive résiduelle dont il s'agit d'évaluer l'importance pour les matières en suspension ou en dilution.

L'expérience est conduite sur la plaque tournante de l'Institut de mécanique de Grenoble, avec pour objectif d'avoir un ordre de grandeur des débits résiduels ainsi induits au large de Boulogne sur Mer. Ils pourraient atteindre $10000 \mathrm{~m}^{3} / \mathrm{s}$, affectant une bande de $10 \mathrm{~km}$ de large avec une vitesse maximale à la côte de l'ordre de $20 \%$ de l'amplitude du courant de marée. Bien entendu, ces résultats ne tiennent pas compte du frottement et font également apparaître des effets tridimensionnels.

La communication de L.HAMM (SOGREA H), J.M. TANGUY (STCPMVN) et B. ZHANG (UTC/GRADIENT) sur la prise en compte des effets gravitaires dans la modélisation du transport solide par charriage s'inscrit dans l'ensemble des travaux menés par le LNH, SOGREAH, le $L H F$, l'UTC et le STCPMVN pour développer un système logiciel de calcul des transports sédimentaires fluviaux et côtiers. Elle constitue un exemple où les nécessités de la modélisation numérique imposent une meilleure connaissance, ou du moins une amélioration des formulations 
traditionnelles, incomplètes, de l'effet de pente. Ce document :

- montre l'importance de la prise en compte des effets gravitaires pour le calcul d'évolutions de fonds mobiles en donnant les résultats de simulations numériques,

- présente une généralisation des formulations existantes pour la prise en compte d' une pente d' angle et de direction quelconques dans le transport solide par charriage.

- fournit une première validation de cette approche sous la forme d'une comparaison intéressante avec les résultats de mesures en laboratoire concernant les évolutions de berges d'un canal dragué dans le sens du courant.

P. PECHON (LNH) s'intéresse à la modélisation mumérique du courant et du transport sédimentaire induit par le déferlement. Il rend compte de travaux effectués dans le cadre des programmes de recherche européens MAST et constituant une recherche en modélisation numérique située en amont des développements de codes opérationnels. Après avoir rappelé les différents types de courants engendrés par la houle au voisinage du littoral, il s'attache à étudier les effets des courants moyens tridimensionnels induits par le déferlement. La simulation des mouvements du fond résulte d'un enchaînement de calculs : propagation de la houle du large vers la côte et déferlement de la houle, courants tridimensionnels engendrés par le déferlement, transport sédimentaire et évolution du fond. Les applications présentées, qui se proposent de valider la modélisation, portent :

- sur deux cas déjà examinés en canaux expérimentaux : circulation dans un canal, formation d'une barre de déferlement.

- sur deux cas schématiques tridimensionnels, afin de vérifier le bon comportement qualitatif du code courantologique et de mettre en évidence les effets tridimensionnels: courant hélicoïdal le long d'une plage rectiligne. courant tridimensionnel derrière un brise-lames.

Les applications à ces cas schématiques donnent des résultats satisfaisants. Mais les travaux apparaissent néanmoins devoir se poursuive dans trois directions : affinement de l'expression des termes figurant dans les équations du modèle de courant, en fonction des caractéristiques locales de la houle, développement d'un modèle de houle sophistiqué, amélioration de l'expression du transport solide en fonction des grandeurs hydrodynamiques.

O. RallLaRd, P. LE HIR et P. LAZURE (IFREMER) ont présenté au colloque des 17 et 18 novembre 1993 une communication sur la mise en place d'un modèle mathématique destiné à rendre compte du transport de sédiments fins dans le bassin de MARENNES-OLERON, premier bassin ostréicole de France. Ce modèle calcule l'évolution spatiale et temporelle des sédiments cohésifs dans la masse d'eau (MES) et sur le fond de vase.

Il cumule de nombreuses et réelles difficultés fondamentales :

- la modélisation des courants est bidimensionnelle. avec des grands estrans et qui jouent un rôle important. - l'agitation engendrée par la houle et le clapot est primordiale dans la mécanique du modèle sédimentologique de vase cohésive.
- la nécessité d'exploiter le calcul sur de longues périodes pour avoir des effets significatifs.

Malgré toutes ces difficultés, les résultats semblent traduire un comportement très encourageant par rapport aux mesures disponibles, avec une surestimation des quantités de vase mises en jeu. La complexité du problème abordé appelle évidemment des approfondissements sur la faşon de mieux prendre en compte la mécanique de la houle et du clapot ainsi que des mesures complémentaires.

Trois communications présentent, enfin, des modèles numériques monodimensionnels de simulation des fonds des rivières.

P. BELLEUDY (LHF) ET H. SCHUTTRUMPF (LIW) décrivent la modélisation de l'évolution morphologique du Damube et de l'Isar. Ils commencent par une brève présentation du système de modélisation SEDICOUP qui réalise la simulation couplée et implicite des écoulements permanents et de l'évolution morphologique du lit d'une rivière, en apportant un soin particulier à l'expression du transport solide en granulométrie étendue et à la modélisation du tri granulométrique et du pavage. Ils donnent ensuite les résultats de la confrontation du modèle avec les données de terrain, sur une section du Danube de quelque $70 \mathrm{~km}$ au voisinage de son confluent avec l'Isar, lui-même modélisé sur quelque $21 \mathrm{~km}$ en amont du confluent. La validation a porté sur la reproduction de l'évolution passée des tronçons de rivière étudiés et sur les évaluations locales de débit solide. La communication se termine par quelques indications sur la poursuite du programme de développement engagé : réflexion méthodologique et extension de ses possibilités.

La communication de T.D. NGUYEN, S.H. LEE, BoIS (INPG) et E. Tormos, Y. GIULIANI (CNR) concerne la modélisation numérique du transport solide en écoulement non permanent et son application à la retenue du BELLEY (Rhône), destinée à prévoir, pour cet aménagement, d'une longueur de $17 \mathrm{~km}$, l'évolution du fond afin d'en déduire les consignes d'exploitation, d'entretien et de transit des crues. Spécialement conçue pour cet aménagement, cette modélisation fait appel à un système d'équations se composant des équations de St Venant, des équations donnant la rugosité du lit, des équations de conservation de la masse du matériau dans la couche de transport en suspension et dans la couche de transport de fond, des équations du débit solide en suspension et en charriage. La confrontation des résultats de la modélisation avec les mesures disponibles s'est révélée satisfaisante, tant au plan hydraulique qu'au plan des dépôts de sédiments en divers points de la retenue.

E. Ben Slama et S. PERon (LNH), P. BELleudy (LHF) et ROUAS (STCPMVN) présentent le code TSAR, modèle monodimensionnel de simulation des évolutions des fonds alluvionnaires des rivières. Fruit d' une collaboration entre le $\mathrm{LNH}$, le $\mathrm{LHF}$, le Ministère de l'Environnement (Direction de l'eau) et le STCPMVN, cet outil vise notamment la simulation, à des échelles de temps variables, des processus complexes de dépôt-érosion et de transport par charriage et suspension de sédiments non cohésifs en granulométrie étendue, tels qu'ils se produisent dans des rivières de plaine soumises à des écoulements subcritiques. 
Après un bref rappel des principaux résultats obtenus lors d'études expérimentales, effectuées en canal, sur le régime permanent du transport par charriage, sans recirculation, de matériaux sableux ou graveleux en granulométrie uniforme ou étendue, la communication présente les spécifications du code TSAR dans sa première version: hypothèses de la modélisation, représentation physique, fermeture, modèle numérique comportant notamment le découplage des phénomènes hydrauliques et sédimentologiques. Elle se termine par le compte rendu de deux tests comportementaux dont les résultats sont très encourageants :

- bief limité par un barrage à l'amont : étude de la passe d'érosion liée au déficit en matériaux à l'aval immédiat du barrage,

- bief limité par un barrage à l'aval : étude du dépôt à l'amont immédiat du barrage qui arrête les sédiments.

\section{ANNEXE - LISTE DES SIGLES UTILISÉS}

CNR Compagnie Nationale du Rhône

IFREMER Institut Français de Recherche pour l'Exploitation de la Mer

INPG Institut National Polytechnique de Grenoble

LHF Laboratoire d'Hydraulique de France

LIW Lichtweiss Institut fur Wasserbau

MAST Marine Science Technology: projets de recherches de la communauté européenne MAST I 1990-1992

MAST II 1993-1995

SOGREAH Société Grenobloise d'Etudes et d'Applications Hydrauliques

STCPMVN Service Technique Central des Ports Maritimes et Voies Navigables

UTC Université de Technologie de Compiègne 